\title{
Design and Implementation of Library Resource Sharing System Database Based on GIS
}

\author{
Hui Pan ${ }^{1}$, Ruixiang Ou ${ }^{1}$, Yao Huang ${ }^{2}$ \\ ${ }^{1}$ Library of South China University of Technology, Guangzhou, 510640, China \\ ${ }^{2}$ Propaganda Department of Southern Medical University, Guangzhou, 510515, China
}

Keywords: GIS, Library resource sharing system, Design and implementation of database

\begin{abstract}
A GIS-based library resource sharing system is designed on account of insufficient resource of individual library and the difficulty of collecting library resources. In the design process of GIS system, the design of database is the core component. This paper takes the library association of universities in Guangzhou University City to conduct a detailed design from the choice of the storage mode of database in the central library, the construction of spatial database and attribute database as well as the correlation and integration of various types of data. The paper proposes the initial implementation of the service function, namely, the system electronic map, thus comprehensively improving the level of library construction and eventually achieving the goal of maximally sharing the information resources.
\end{abstract}

\section{Introduction}

GIS (Geographic Information System) was first known in the 1960s, which is a geospatial technology and interdisciplinary science developing rapidly, which employs spatial data and attribute data in analyzing and processing data. In addition, it links the two together to have common management, analysis and application through the database management system so as to provide a new thinking mode. The development of GIS technology has fundamentally changed the way of obtaining, sorting and retrieving data. Previous researches carried out by scholars abroad have fully proven the necessity and feasibility to introduce GIS in library management. However, the previous researches served the individual library as study object to prospect the prospect functions and goals of the system, and applying the GIS into library resource sharing and planning decision is deficient in combination with Internet technology and Web browser. With the reference to the related research results of GIS in other fields, this paper profoundly explores how to use GIS to innovate the library resource organization mode and service management mode in the information construction process of libraries.

The introduction of GIS to the information construction of libraries can realize the following main features. First of all, visual and unified search on literature resources in the region can be achieved. Secondly, it can measure the distance and traffic routes to the library by employing the GIS measuring function. In addition, based on the data analysis function of GIS, the book situation inside the attribute database and borrow situation of literature resources and so on can be collected and analyzed, thus further forecasting the rational distribution and allocation of the library information resources in the geographical area. According to the results, cross-system and cross-sectorial cooperation and procurement can be carried out so as to play the comprehensive strength after cooperation and sharing among different types of libraries, thus achieving complementary advantages and promoting the overall development of the library industry.

The design of database is the core component of GIS system design. The database will have a variety of organizational forms according to different GIS applications. The paper takes the construction of GIS-based library resource sharing system as an example to mainly probe into the design of database. Combined with system requirements and applications, the paper has a detailed design from the choice of data storage mode, construction of spatial database and attributes database as well as the association and integration of various data. Finally, the paper expounds the initial realization of library resource sharing system. 


\section{Design of Library Resource Sharing System Database}

In this paper, it takes the uses of GIS, Relational Database Management System (RDBMS) and Computer Network Technology (CNT) to complete the design of GIS-based regional resource database in libraries. While in the database layer, it employs distributed technology to gradually refine the hierarchical management of libraries in the region, surrounding traffic and other data on spatial location in order to establish a spatial database, and then associated with established library information. Dynamically integrating the information distributed in different libraries together through the Internet protocol to can provide users with a unified visual environment for retrieving information, thus further enhancing the levels including sharing and exchange of library information, comprehensive utilization and socialized services.

System Structure. The regional library sharing system uses a star-shaped radiation structure. In other words, it establishes a central library as the core leader apart from the member libraries. According to the design objectives of this system and the characteristics of various types of data, the data storage in the central library selects the relational database management mode whose basic idea is to add a layer of spatial database engine based on the conventional database management system to obtain the data storage and management capabilities in addition to the functions of regular database management system. The data of member libraries depends on the real situation of different libraries, which are suggested to be reunified with the central library in order to facilitate association among data.

The design of system central library database employs integrated management approach between ArcSDE spatial database engine and SQL Server2000 database, the latter in which provides complete functions for data management and backup and recovery, supporting distributed architecture for multi-servers. By contrast, ArcSDE extends the powerful data management capability of RDBMS from non-spatial data to spatial data. In consequence, managers can build massive, multi-user-based database systems to accept the users' concurrent accesses so that meet the sharing for users on information resources.

Data Organization. Before constructing the database, it is noted to collect and organize all the required data first of all. The data involved in the GIS is different from that in the conventional management information, which is a collection of geographic data possessed with abundant geographical features. The data is featured with large information content. In addition, the accesses and sources between the spatial information data and non-spatial information data are totally different. According to the construction program of this system database, the data can be divided into spatial data and business data, the former one of which mainly refers to graphic data. Sometime, the system operation requires the support of spatial data, while only needs to manage a large number of business data occasionally, which is free of the operations such as the display of spatial data. In order to improve the response speed of the system, the business data of the system is mostly stored in the database of every individual library.

In accordance with the analysis on the functional requirements and system target of the system, it raises a high demand for real-time data. The map data adopted by the system is supposed to conform to the actual situation. In contrast, the system calls a comparatively low claim for the mathematical precision. This paper chooses five road intersections as measurement control points of GPS and takes the obtained data from GPS as control point data to register the map under ARGIS software. Based on the digital content and analysis of system goals, this paper takes ARCVIEW3.2 to digitize the screen so as to store each university library as a thematic layer, thus finally generating multiple files of SHAPE format and then supplementing necessary information for several property fields in the various layer designs. The design of the attribute database in this system includes two types of attribute tables, one of which is the attribute table of business data and the other is of graphic elements. For the former one, the system has designed the administrator information table, the reader information table, library information table, borrow information table, fine information table, system maintenance information table, user rights information table and so on so forth. It expresses the connection among these concepts as well. 
Association between Spatial Attribute Data and Business Data. The part of the attribute data stored in the spatial database only describes some basic information (such as library name, ISBN number, etc.) of the graphic data. The specific and frequently changed business data is stored in table in the database independently, and there's often no graphical information to match it directly. The system design goal is required to inquire about all kinds of library information (including library introduction, document resource information, reader borrowing information and traffic information of the museum) in the shared network as well as the statistical analysis function of the information. The implementation of these functions requires that the spatial attribute data be associated with the business data. After establishing the connection between spatial attribute data and business data, it can be easy to search corresponding business data through spatial graphics data and find out corresponding spatial graphics data through business data. What's more, business data can be presented by making a dynamic and thematic statistical chart based on the dynamic change of the business data.

The premise of the association between graphical attribute data and business data is that the graphical attribute data is used for the associated field to ensure its uniqueness and cannot be null, and there is a common field between the two, for example, The field "Lib" in the attribute data table of the library layer with point feature and the field "holding library" in the document resource information table can satisfy these conditions. This association is only a logical association, not a physical one, that is, a temporary association. After that, logically, the business data is added to the tail of the graphic attribute data to the corresponding field, and the business data can be accessed through the attribute data of the layer.

\section{Initial Implementation of Library Resource Sharing System}

The Design and Implementation of the Main Interface in System. Compared with the traditional library automation system, map display and visual query are highlights and core functions of the system. When designing the main interface, the electronic map of the study area is set as the main window, furthermore, the web interface also includes the title area, user authentication, function navigation items, links of each pavilion and so on.

In terms of the retrieval system, the book name, publication year, publishing, sorting and other search conditions are provided. After obtaining system retrieval results in the client, users can click on the book of interest, query the specific collection directory, and obtain the system collection location, and even the system can also query the route in the geographical display. After obtaining the detailed address of the book, the system will provide a simulation bookshelf system to better show the detailed address of the book. The system will also display the specific floor which the book is located after getting the specific library information. When the user clicks on the link, the system will stimulate the specific floor map, which greatly reduces the user's retrieval time. Then, the system will simulate the specific location of the system for the user to accurately locate the system location. Flickering with red dots prompts the user for precise positioning. At the same time, the system supports drag and drop conversion to view the specific address of the book, or even enlarge the basic information to see books.

The Development and Implementation of Map Service Function. ArcIMS is a kind of software that creates and maintains a geodata Website. After a series of edits in ArcView, ArcIMS can create a website through ArcIMS. It provides users with a powerful map data browsing, customization and publishing capabilities through an easy-to-use framework. First ArcIMS Author is used to complete the map configuration. After generating all map configuration files, you can use ArcIMS Administrator to start the map service of the release site. Beyond the above, you can complete the prototype creation of the website through a very simple wizard Dialog box that provided by ArcIMS Designer tool, and then generate the web service for users.

Once the customization and development work is complete, the system can be published as an official website. IE browser can be used as landing site for browsing and query operation. Library sharing system provides a joint bibliographic query system and electronic resource joint retrieval 
system, both of which achieve the retrieval of literature resources. For the collection of printed literature resources, we mostly use MARC for a unified search. The bibliographic database is self-built by each pavilion, and they can download standard MARC data from CALIS for cataloging data sharing. All kinds of electronic resources system provides electronic resources joint retrieval system, which aims to integrate a variety of heterogeneous digital resources and provide users with a better integrated search services, thereby enhancing the utilization of resources. The user first visit the GIS system Website through the browser, and enter the query interface to input keywords to form a suitable search to express users' retrieval needs, the system will form search results in table according to the users' search needs, and return to them.

The search result set mainly shows the basic situation and the collection place of all the literature information which satisfies the search type. The user can enter the map service function interface by clicking on any of the collection places in the result set, and then click the highlight in the electronic map to satisfy the retrieval condition Collection, that is, pop up the museum's attribute information, and measure the distance and traffic routes between the self-location and collection by GIS so as to choose the best way of borrowing.

In the system development process, HTML Viewer template customization can only achieve the basic, simple spatial query function. In order to realize the more complex spatial query and statistical analysis function of GIS, it is necessary to extend the system function by connecting the external business attribute data through the key field.

\section{Conclusion}

Based on the research of GIS service in domestic and foreign libraries, the paper takes the library of Guangzhou University as the research object according to the present situation and management practice of library in China, and makes a detailed design of the database system of library sharing system in combination with GIS technology, And some key technologies of system database construction are researched and explored deeply. The whole-relational database management method of graphic data and attribute data can be realized, and then it can be combined with $\mathrm{B} / \mathrm{S}$ architecture to make use of ArcIMS online Release platform to initially realize the electronic map service function.

\section{Acknowledgements}

This paper is one of the research results of "On Literature Resources Sharing System of Library Associations of in Universities of Guangdong Province Based on GIS” (Item No. GD14XTS04), which is the "Philosophy and Social Science" project during the Twelfth Five-year Plan Period in 2014.

\section{References}

[1] Zhang Linna, Hu Yuan, Liang Wei, Research and Application of the Library Space Management System Based on 2.5 dimensional GIS [J]. New Century Library, 2014(9): 52-55.

[2] Xia Yujie, Zhai Yanlei, Design and implementation of embedded GIS map navigation based on Mitab library [J]. Computer Engineering and Design, 2011, 32(4): 1303-1306.

[3] Liu Li, Xia Youbin, Design and Implementation of Database in Library Sharing System Based on GIS [J]. Library and Information Service, 2013, 57(19): 97-101.

[4] Yang Mai, Wang Xuemei, A Bibliometrics Analysis of World Libraries Papers based on GIS [J]. Remote Sensing Technology and Application, 2015, 30(4): 819-824. 